\title{
The relativistic virial theorem and scale invariance
}

\author{
José Gaite \\ IDR, Universidad Politécnica de Madrid, E-28040 Madrid, Spain
}

May 29, 2013

\begin{abstract}
The virial theorem is related to the dilatation properties of bound states. This is realized, in particular, by the Landau-Lifshitz formulation of the relativistic virial theorem, in terms of the trace of the energy-momentum tensor. We construct a Hamiltonian formulation of dilatations in which the relativistic virial theorem naturally arises as the condition of stability against dilatations. A bound state becomes scale invariant in the ultrarelativistic limit, in which its energy vanishes. However, for very relativistic bound states, scale invariance is broken by quantum effects and the virial theorem must include the energy-momentum tensor trace anomaly. This quantum field theory virial theorem is directly related to the Callan-Symanzik equations. The virial theorem is applied to QED and then to QCD, focusing on the bag model of hadrons. In massless QCD, according to the virial theorem, 3/4 of a hadron mass corresponds to quarks and gluons and $1 / 4$ to the trace anomaly.
\end{abstract}

PACS: 03.30.+p, 11.10.St, 12.38.Aw, 12.39.Ba

\section{Introduction}

The classic virial theorem has been very useful in physics, in particular, in astrophysics, to determine the equilibrium and stability of dynamical systems [1]. The theorem is especially useful for systems interacting through potentials that are homogeneous functions of the interparticle distances (e.g., power law potentials): then the theorem states a simple relation between the long-time averages of the kinetic and potential energies of a system, namely, $2 K=n U$, where $n$ is the homogeneity degree (e.g., the exponent of the power law). Of course, the most relevant potentials to consider are the quadratic potential of harmonic oscillations $(n=2)$ and the inverse distance law of the Newton or Coulomb potentials $(n=-1)$. The virial theorem for homogeneous potentials can be regarded as a consequence of mechanical similarity, in other words, of scale invariance in mechanics [2]. Indeed, several authors have studied the relation of the virial theorem to scale invariance $[3,4,5]$ and its connection with Noether's theorem $[6,7,8]$. 
The classic virial theorem has been generalized in several ways [1]. The obvious generalizations pertain to relativistic mechanics and quantum mechanics. Whereas the generalization of the virial theorem to non-relativistic quantum mechanics presents no special problems, the generalization to relativity is non-trivial, because the concepts of force and potential are unsuitable for describing relativistic interactions. Notwithstanding, there are relativistic formulations of the virial theorem. A virial theorem in electrodynamics that expresses the energy in terms of the energy-momentum tensor trace already appears in the classic textbook by Landau and Lifshitz [9]. This relativistic virial theorem is generalizable, in principle, to other interactions [1].

The relativistic virial theorem has featured in several works and some papers have been specifically devoted to it, both in classical field theory $[10,11]$ and in quantum field theory [12]. However, while the relation of the classic virial theorem to scale invariance is well established, the relation to scale invariance of the relativistic virial theorem, especially, in the Landau-Lifshitz formulation, is hardly studied. In this paper, we focus on this relation and adopt a fundamental standpoint. Therefore, our approach to the relativistic virial theorem is closer to the one of Ref. [11] and especially to the one of Ref. [12] than to the one of Ref. [10]. Our main purpose is to establish the fundamental role of scale transformations and scale invariance in the relativistic virial theorem. For this purpose, we find it useful to proceed from the classic theorem to the relativistic theorem and, finally, to the virial theorem in quantum field theory (QFT). In this process, the classic theorem $2 K=n U$, which allows for any homogeneity degree $n$, must be restricted to relativistic interactions mediated by a field that becomes in the non-relativistic limit just an inverselaw potential, so the only allowed value is $n=-1$. In other words, the virial theorem is restricted to interactions mediated by massless gauge fields, namely, the electromagnetic interaction and the strong interaction described by quantum chromodynamics (QCD). The latter has special features, as will be explained.

The classic virial theorem includes the gravitational interaction, but the relativistic formulation of gravity necessarily leads to General Relativity, which is a sort of interaction mediated by a massless gauge field, although not of the ordinary type. In fact, there have been attempts to formulate the virial theorem in General Relativity [1, p 27], but they neglect its relation to other gauge theories. Some of the results of the present paper can be applied to General Relativity, but this theory has distinct features and, in particular, in it the concept of gravitational energy as well as the concept of scale invariance become very subtle and difficult to handle. These problems are beyond the scope of this paper and are left for future work.

We begin in Sec. 2 with a Hamiltonian formulation of the virial theorem that directly arises from the notion of time-averaged scale invariance. This formulation is applied to the electromagnetic interaction in special relativity, considering, first, the action-at-a-distance interaction of particles and, second, the full field theory, following Landau and Lifshitz [9]. Next, in Sec. 2.2, we construct a fully general derivation of the field theory virial theorem that is based on scale invariance, by generalizing the Hamiltonian formulation of the virial theorem for a system of particles. Some consequences of the virial theorem for bound states of ultrarelativistic particles appear in Sec. 2.3. As these bound states sustain considerable 
quantum effects, they call for relativistic quantum mechanics. The quantum field theory virial theorem is studied in Sec. 3, applying it, first, to quantum electrodynamics (QED) and, second, to quantum chromodynamics (QCD), in which the energy-momentum tensor trace anomaly plays a crucial role.

\section{The relativistic virial theorem}

The virial theorem is regarded by Landau and Lifshitz [2] as a consequence of scaling in Lagrangian mechanics. This line of thought has been followed by several authors $[3,4,5,8]$, some of whom relate the virial theorem to the Noether theorem. In contrast to Lagrangian scaling, we now introduce the general theory of dilatations in the Hamiltonian formalism, which provides a concise and powerful formulation of the virial theorem.

In Hamiltonian mechanics, canonical transformations are the most general transformations that preserve the phase space structure [2]. A canonical transformation of the phase space $(q, p)$ is defined by its infinitesimal generator, which is just any function $F(q, p)$. For simplicity, we use single variables $q$ and $p$, but each denotes the full set of coordinates or momenta. The transformed phase space variables are:

$$
\begin{gathered}
Q=q+\delta_{F} q, \quad \delta_{F} q=\{q, F\}=\frac{\partial F}{\partial p}, \\
P=p+\delta_{F} p, \quad \delta_{F} p=\{p, F\}=-\frac{\partial F}{\partial q},
\end{gathered}
$$

where we have introduced Poisson brackets $\{\cdot, \cdot\}$. Naturally, the phase-space volume element $d q d p$ is unchanged. Among canonical transformations, an important role is played by point transformations, namely, canonical transformations induced by transformations of the coordinates $q$ only. They are generated by $F=f(q) p$, where $f$ is any function. If $F=q p$, we have

$$
\delta_{F} q=q, \quad \delta_{F} p=-p,
$$

that is, a homogeneous dilatation of $q$ and the corresponding homogeneous contraction of $p$, which together preserve the phase space volume. But, normally, the dilatation of $q$ is not a symmetry, that is,

$$
\delta_{F} H=\{H, F\}=q \frac{\partial H}{\partial q}-p \frac{\partial H}{\partial p} \neq 0,
$$

and $F$ is not a constant of the motion, that is, $\dot{F}=\{F, H\} \neq 0$, unless the Hamiltonian $H$ is very special (e.g., $H=F$ ). Nevertheless, if both $q$ and $p$ are bounded, the temporal average of $\dot{F}$ vanishes in the long run, so that, on the average,

$$
\{F, H\}=p \dot{q}-q \frac{\partial H}{\partial q}=H+L-q \frac{\partial H}{\partial q}=0 .
$$

Note that the coordinates $q$ of a system of particles can only be bounded in their rest frame, namely, the frame in which their total momentum is zero. 
In many mechanical problems, $L(q, \dot{q})=K(\dot{q})-U(q)$, namely, there is a separation between kinetic and potential energies, and $K$, in addition, is quadratic in the velocities. Then, $H=K+U$, so

$$
H+L-q \frac{\partial H}{\partial q}=2 K-q \frac{\partial U}{\partial q}=0 .
$$

The term $q \partial_{q} U$ is called virial. Furthermore, when $U(q)$ is a homogeneous functions of $q$ of degree $n$, Euler's theorem on homogeneous functions implies that $q \partial_{q} U=n U$, so there follows the standard virial theorem, $2 K=n U$ or, in terms of the total energy $E$, $K=n E /(n+2)[1,2]$. This theorem is a consequence of the homogeneity of $K$ in $\dot{q}$ and the homogeneity of $U$ in $q$, which also imply mechanical similarity: the equations of motion permit a set of geometrically similar motions, such that the paths are geometrically similar and the times of motion between corresponding points are in a constant ratio [2]. For example, when $n=-1$, as in the Newton or Coulomb potentials, to $q \rightarrow l q$ correspond $t \rightarrow l^{3 / 2} t$ (Kepler's third law) and $E \rightarrow E / l$.

Unfortunately, the Lagrangian in relativistic mechanics is not of the form $L(q, \dot{q})=$ $K(\dot{q})-U(q)$ with $K$ homogeneous in $\dot{q}$. Nevertheless, the classical virial theorem is easily extended to the case of a relativistic particle under external forces [13]. Moreover, the Hamiltonian virial theorem, Eq. (2), is very general and covers the many-body problem in relativistic electrodynamics, as the prototype of relativistic interactions. Before considering this problem, let us consider two pertinent extensions of the Hamiltonian virial theorem. The first one is the extension to quantum mechanics. This extension is straightforward, because the Hamiltonian formulation of canonical transformations is easily transferred to quantum mechanics, just by transforming Poisson brackets to commutators. However, one has to take care of operator ordering problems, for example, by symmetrizing phase space functions with respect to $q$ and $p$. Any phase space function that can be expanded in powers of $q$ and $p$ can be symmetrized by symmetrizing every term of the expansion (which is a polynomial). In particular, the dilatation generator becomes $F=(q p+p q) / 2$ (naturally, $p=-i \hbar \partial / \partial q$, in the coordinate representation).

As a second extension of the virial theorem, this theorem can also be extended to canonical transformations other than dilatations. Indeed, the long-time average of $\dot{F}$ vanishes for any bounded function $F$ and so does the average of $\{F, H\}$. Therefore, we have an infinite number of (average) relations. However, general canonical transformations, with generators $F$ that depend on $p$ arbitrarily, have little physical significance. In contrast, $F=f(q) p$, namely, the set of point transformations, includes rotations and, furthermore, arbitrary deformations of the geometrical "shape" of the mechanical system. These transformations give rise to an extension of the standard virial theorem, namely, the tensor virial theorem [1], to be discussed further in page 7 . 


\subsection{Virial theorem for the electromagnetic interaction}

The $N$-particle electromagnetic Lagrangian is $[9,14]$

$$
L=\sum_{a=1}^{N}\left(-m_{a} c^{2} \sqrt{1-\frac{\boldsymbol{v}_{a}^{2}}{c^{2}}}+\frac{e_{a}}{c} \boldsymbol{v}_{a} \cdot \boldsymbol{A}\left(\boldsymbol{x}_{1}, \ldots, \boldsymbol{x}_{N}\right)-e_{a} \Phi\left(\boldsymbol{x}_{1}, \ldots, \boldsymbol{x}_{N}\right)\right),
$$

where $\boldsymbol{v}_{a}=\dot{\boldsymbol{x}}_{a}$. The corresponding Hamiltonian is

$$
H=\sum_{a=1}^{N}\left(\sqrt{\left(c \boldsymbol{p}_{a}-e_{a} \boldsymbol{A}\right)^{2}+m_{a}^{2} c^{4}}+e_{a} \Phi\right) .
$$

To allow for interaction between the particles, the electromagnetic potentials must fulfill the D'Alembert wave equation with a source given by the electromagnetic current produced by the particles themselves, so the potentials are functions of the particle trajectories. If the symmetric Green's function (half-sum of advanced and retarded functions) is used to solve the wave equation, the Lagrangian or Hamiltonian correspond to action-at-adistance electrodynamics $[15,16]$, in which there is no radiation and, therefore, no need of the electromagnetic field Lagrangian.

Neither the $N$-particle Lagrangian nor the Hamiltonian can be separated into kinetic and potential terms, but we can apply Eq. (2) nonetheless. Given that

$$
\left(\frac{\partial H}{\partial \boldsymbol{x}_{a}}\right)_{\boldsymbol{p}}=-\dot{\boldsymbol{p}}_{a}=-\left(\frac{\partial L}{\partial \boldsymbol{x}_{a}}\right)_{\boldsymbol{v}}
$$

Eq. (2) writes

$$
\begin{array}{r}
H+L+\sum_{a} \boldsymbol{x}_{a} \cdot \frac{\partial}{\partial \boldsymbol{x}_{a}}\left(\sum_{b} \frac{e_{b}}{c} \boldsymbol{v}_{b} \cdot \boldsymbol{A}-e_{b} \Phi\right)= \\
H+\sum_{a}\left[-m_{a} c^{2} \sqrt{1-\frac{\boldsymbol{v}_{a}^{2}}{c^{2}}}+\left(1+\boldsymbol{x}_{a} \cdot \frac{\partial}{\partial \boldsymbol{x}_{a}}\right)\left(\sum_{b} \frac{e_{b}}{c} \boldsymbol{v}_{b} \cdot \boldsymbol{A}-e_{b} \Phi\right)\right]=0
\end{array}
$$

where the derivatives are to be taken for constant $\boldsymbol{v}_{b}$. Notice that the action of the operator $\sum_{a}\left(1+\boldsymbol{x}_{a} \cdot \partial / \partial \boldsymbol{x}_{a}\right)$ on a homogeneous function of $\boldsymbol{x}_{1}, \ldots, \boldsymbol{x}_{N}$ of degree $n=-1$ gives a null result, according to Euler's theorem. In other words, if we assume that the vector and scalar potentials can be written as homogenous functions of the coordinates with degree $n=-1$, each electromagnetic potential and its corresponding virial cancel one another. So we obtain the energy

$$
E=\sum_{a} m_{a} c^{2} \sqrt{1-\frac{\boldsymbol{v}_{a}^{2}}{c^{2}}}
$$

This is the relativistic virial theorem for a system of particles in classical electrodynamics. If external forces were necessary to confine the particles and, in particular, the forces 
consisted in a constant pressure $P$ exerted on the system's surface, then $E$ should be replaced with $E-3 P V$, where $V$ is the system's volume $[9, \S 35]$.

Although the electromagnetic parameters are absent from Eq. (5), their effect is implicitly included: notice that $E<\sum_{a} m_{a} c^{2}$, as corresponds to a bound state. The bound state becomes non-relativistic for low velocities, when $\left|E-\sum_{a} m_{a} c^{2}\right| \ll \sum_{a} m_{a} c^{2}$ and Eq. (5) reduces to the classical virial theorem $E-\sum_{a} m_{a} c^{2} \approx-\sum_{a} m_{a} v_{a}^{2} / 2=-K$. However, the relativistic dynamics has lost the similarity of the classical dynamics under space dilatations and corresponding time dilatations. This similarity is lost even assuming that $\Phi$ and $\boldsymbol{A}$ are homogenous functions with degree $n=-1$. Moreover, in relativistic mechanics, there is no similarity even for free particles $\left(e_{a}=0\right)$, because of the form of the Hamiltonian. (Of course, similarity is recovered for low velocities, but the energy only scales after subtracting the particles' rest energy $\sum_{a} m_{a} c^{2}$.) However, there is a relativistic notion of mechanical similarity [4], in which space and time are equally dilatated, so velocities are unchanged. Under this similarity, masses cannot be held constant and must be contracted so that they transform like energies. Therefore, the nature of the particles is changed and the similarity does not relate different motions of the same system. Nevertheless, this similarity leads to Eq. (5) and, in addition, it also leads to the classic virial theorem in the non-relativistic limit [4].

The equations of motion of action-at-a-distance electrodynamics are not ordinary differential equations but differential-difference equations, and it is hard to find solutions of them. A simple solution of the relativistic two-body problem is provided by two opposite charges in circular motion, with calculable radii and angular velocity [15, p. 223]. This solution fulfills Eq. (5) (no temporal average is needed). At any rate, it is natural to consider the electromagnetic field's dynamics and the radiation effects. However, given that these effects appear, in an expansion in powers of $v / c$, only in the third order, it is possible to describe the electromagnetic interaction of particles with standard ordinary differential equations based on potentials of order $(v / c)^{2}[9,14,15]$. These potentials, added to a relativistic kinetic term expanded to the same order, give rise to the well-known Darwin Hamiltonian and Lagrangian. In this approximation, both the vector and scalar potentials are homogeneous functions of degree $n=-1$, so the virial theorem (5), expanded to terms of second order, holds.

Regarding quantum mechanics, the relativistic virial theorem holds, by canonical quantization of Hamiltonian systems, as said before. Indeed, quantum relativistic versions of the virial theorem have appeared in the literature $[17,18]$. However, they are meant to be applied in nuclear physics and they only consider a simple two-quark problem with a phenomenological scalar potential $U$ (notice that the fundamental theory of strong interactions, namely, QCD, includes a vector potential, like QED). The scalar potential is the "Cornell potential," which is the sum of a Coulomb term and a confining linear term. Lucha and Schöberl [17], in particular, obtain

$$
\langle\boldsymbol{x} \cdot \nabla U(\boldsymbol{x})\rangle=c\left\langle\frac{\boldsymbol{p}^{2}}{\sqrt{\boldsymbol{p}^{2}+\left(m_{1} c\right)^{2}}}+\frac{\boldsymbol{p}^{2}}{\sqrt{\boldsymbol{p}^{2}+\left(m_{2} c\right)^{2}}}\right\rangle,
$$


and, hence,

$$
E=\langle\boldsymbol{x} \cdot \nabla U(\boldsymbol{x})\rangle+\langle U(\boldsymbol{x})\rangle+c^{3}\left\langle\frac{m_{1}^{2}}{\sqrt{\boldsymbol{p}^{2}+\left(m_{1} c\right)^{2}}}+\frac{m_{2}^{2}}{\sqrt{\boldsymbol{p}^{2}+\left(m_{2} c\right)^{2}}}\right\rangle,
$$

where the expectations values are understood to be taken with respect to normalized eigenstates. Eq. (6) states the equality of the expectations values of the centripetal and centrifugal virials. On the other hand, on account of the relativistic identity

$$
1-\frac{\boldsymbol{v}^{2}}{c^{2}}=\frac{(m c)^{2}}{\boldsymbol{p}^{2}+(m c)^{2}}
$$

Eq. (7) is a quantum version of Eq. (4), such that the temporal expectation values implicit in Eq. (4) are replaced by expectation values on stationary states and such that the system is restricted to two particles with $\boldsymbol{A}=0$. In Eq. (7), the Coulomb part of the potential and its virial indeed cancel one another, like in Eq. (4). At any rate, the strong interaction Hamiltonian employed by Refs. $[17,18]$ is not fully relativistic and only has relativistic kinematics (the potential $U$ can be interpreted as the lowest order slow-motion approximation of a relativistic interaction). The formulation of a fully relativistic virial theorem for quark bound states requires a QFT framework and is presented in Sec. 3.

Returning to classical electrodynamics, let us consider the full particle plus field dynamics and its local conservation laws, namely, the local conservation of energy and momentum. Landau and Lifshitz's virial theorem [9] relies on this conservation law, expressed in terms of the energy-momentum tensor. This conservation law implies that the long-time average of the stress tensor (the spatial part of the energy-momentum tensor) is divergenceless; that is,

$$
\partial_{j} \overline{T_{i}^{j}}=0,
$$

where Latin indices denote spatial coordinates and the bar denotes the long-time average. Multiplying Eq. (8) by $x^{i}$ and integrating over all space, one obtains (under suitable asymptotic conditions) that the space integral of the stress-tensor trace average vanishes:

$$
\int \overline{T_{i}^{i}} d V=0
$$

Actually, the vanishing of the integral of the full stress tensor for a closed (self-contained) and static system was proved by Laue [19] at the beginning of relativity theory. ${ }^{1}$ The vanishing of the integral of the time average of the stress tensor constitutes the tensor virial theorem, which holds in classical mechanics as well as in relativistic mechanics (Ref. [1], $\S$ II.1 and $\S$ II.3). It can be proved by multiplying Eq. (8) by $x^{k}$, that is, using a generic index $k$ instead of the index $i$ used above, and integrating over all space. Regarding the connection of the tensor virial theorem with arbitrary transformations of spatial coordinates, already

\footnotetext{
${ }^{1}$ Laue's paper studies the energy and momentum of a closed static system and, according to Ohanian [20], it contains the first real proof of the mass-energy equivalence, $E=m c^{2}$.
} 
mentioned in page 4, this theorem can be understood as just a condition of dynamical equilibrium, namely, of stability of the averaged system shape against deformations, as explained in Sec. 2.2. Of course, the tensor virial theorem implies the scalar theorem (the vanishing of the trace), which can be understood as a condition of stability of the system against dilatations.

For a system of $N$ bodies in electromagnetic interaction,

$$
T_{i}^{i}=\sum_{a=1}^{N} \frac{m_{a} \boldsymbol{v}_{a}^{2}}{\sqrt{1-\boldsymbol{v}_{a}^{2} / c^{2}}} \delta\left(\boldsymbol{x}-\boldsymbol{x}_{a}\right)+\frac{1}{2}\left(\boldsymbol{E}^{2}+c^{2} \boldsymbol{B}^{2}\right) .
$$

Therefore, the space integral of $T_{i}^{i}$ seems to be strictly positive and the virial theorem, Eq. (9), cannot be fulfilled. To interpret Eq. (10) and the corresponding virial theorem, let us make the orthogonal decomposition $\boldsymbol{E}=\boldsymbol{E}_{\mathrm{L}}+\boldsymbol{E}_{\mathrm{T}}$, where $\boldsymbol{E}_{\mathrm{L}}=-\nabla \Phi$ and $\nabla \cdot \boldsymbol{E}_{\mathrm{T}}=0$. Then, the electromagnetic energy writes

$$
\frac{1}{2} \int d V\left(\boldsymbol{E}^{2}+c^{2} \boldsymbol{B}^{2}\right)=\frac{1}{2} \int d V\left(\boldsymbol{E}_{\mathrm{L}}^{2}+\boldsymbol{E}_{\mathrm{T}}^{2}+c^{2} \boldsymbol{B}^{2}\right)
$$

where

$$
\frac{1}{2} \int d V \boldsymbol{E}_{\mathrm{L}}^{2}=\frac{1}{8 \pi} \int d V d V^{\prime} \frac{\rho(\boldsymbol{x}) \rho\left(\boldsymbol{x}^{\prime}\right)}{\left|\boldsymbol{x}-\boldsymbol{x}^{\prime}\right|}
$$

is the Coulomb electrostatic energy. While this electrostatic energy is obviously positive for regular distributions, the electrostatic energy of a system of $N$ positive and negative point-like charges can become negative after the subtraction of the (infinite) self-energy of the charges, which amounts to a renormalization of their masses $[9,14]$. On the other hand, regarding the contribution of $\boldsymbol{E}_{\mathrm{T}}$ and $\boldsymbol{B}$ to the energy, one must impose the absence of incoming or outgoing radiation fields, which will produce contributions to $\boldsymbol{E}_{\mathrm{T}}^{2}+c^{2} \boldsymbol{B}^{2}$ of arbitrary magnitude. Indeed, the proof of Eq. (9) requires the vanishing of certain surface integral related to radiation fields [9]. As already said, the absence of radiation is implicit in action-at-a-distance electrodynamics. These problems have already been noticed by Dudas and Pirjol [12], who emphasize the role of the Wheeler-Feynman time-symmetric formulation of electrodynamics in their solution.

The four-dimensional trace is $T_{\mu}^{\mu}=-T^{00}+T_{i}^{i}$ [our metric's signature convention is $(-,+,+,+)]$. Therefore, a condition equivalent to $(9)$ that introduces the total energy is $[9]$

$$
E=-\int T_{\mu}^{\mu} d V
$$

(like before, the long-time average is implicit when not explicitly shown). For a system of electromagnetically interacting particles, the electromagnetic energy-momentum tensor is traceless and disappears from Eq. (13). This absence of electromagnetic parameters at virial equilibrium is analogous to the cancellation of the electromagnetic potentials with their corresponding virials in Eq. (4). Indeed, Eq. (13) leads again to Eq. (5) [9].

Notice that Eq. (13) makes no reference to particles, unlike Eq. (5). Therefore, it can be applied to fields forming a single particle and then it plays a role in the famous 
problem of modeling the electron or any charged particle in classical electrodynamics [14, Ch. 16]. Indeed, Eq. (13) connects the particle's energy with the trace of the energymomentum tensor of the Poincaré stresses, since the electromagnetic energy-momentum tensor is traceless. If we assume, for simplicity, that the energy-momentum tensor of the Poincaré stresses has no traceless part, namely, that it is proportional to $g_{\mu \nu}$, then the total energy-momentum tensor fulfills $T^{\mu \nu}=T_{\mathrm{em}}^{\mu \nu}+T_{\alpha}^{\alpha} g^{\mu \nu} / 4$, where $T_{\mathrm{em}}^{\mu \nu}$ is the energy-momentum tensor of the electromagnetic field. Therefore, in the rest frame,

$$
E=\int\left(T_{\mathrm{em}}^{00}+\frac{g^{00}}{4} T_{\alpha}^{\alpha}\right) d V=\int T_{\mathrm{em}}^{00} d V+\frac{E}{4},
$$

so three fourths of the particle's rest energy come from its electromagnetic energy and the remaining one fourth from the Poincaré stresses (3/4 is actually the ratio of electromagnetic mass to electromagnetic inertia that constitutes the infamous $4 / 3$ problem, solved by the introduction of the Poincaré stresses [14, Ch. 16]). If the energy-momentum tensor corresponding to the Poincaré stresses, $T_{\mathrm{P}}^{\mu \nu}$, has a non-vanishing traceless part, this part is on the same footing as the traceless energy-momentum tensor of the electromagnetic field, so both together make up the three fourths of the total particle's rest energy. The contribution to the energy of the traceless part of the energy-momentum tensor for the Poincaré stresses is the space integral of

$$
T_{\mathrm{P}}^{00}-T_{\mathrm{P} \alpha}^{\alpha} \frac{g^{00}}{4}=\frac{3}{4} T_{\mathrm{P}}^{00}+\frac{1}{4} T_{\mathrm{P}_{i}^{i}}^{i} .
$$

This integral is non-negative, despite that $\int T_{\mathrm{P}_{i}^{i}}^{i} d V<0$, as is necessary to have cohesive Poincaré stresses, that is to say, as is necessary for the total stress tensor to fulfill Eq. (9). The non-negativity of the right-hand side of Eq. (15) can be proved by invoking the null energy condition (deduced by continuity from the non-negativity of the energy density [21, p. 89]). In conclusion, the proportion of energy due to the Poincare stresses is at least one fourth, and the proportion of electromagnetic energy can be equal or smaller than three fourths.

In a single-particle model, the charge distribution is continuous and can be attributed to a charged field or fluid. An interesting classical electron model is Bialynicki-Birula's [22], in which the electron consists of a perfect, charged fluid, with energy density $\rho$ and pressure $P$, and the electromagnetic field. The traceless part of the fluid energy-momentum tensor is proportional to $\rho+P$ and contributes to the energy with $(3 / 4) \int(\rho+P) d V$, which is non-negative, despite that $P<0$ everywhere.

Notice that a particle model with a continuous charge distribution makes as much sense for a composite particle as for an elementary particle, since there is no quantization of charge in classical electrodynamics. For consistency with electron modeling in QED, let us assume that matter is described by a Dirac field $\psi$ with the standard Lagrangian. Then,

$$
T_{\mu \nu}=\frac{i}{2} c\left(\bar{\psi} \gamma_{(\mu} \mathcal{D}_{\nu)} \psi-\mathcal{D}_{(\mu}^{*} \bar{\psi} \gamma_{\nu)} \psi\right),
$$


where $\mathcal{D}_{\mu}=\hbar \partial_{\mu}+i(e / c) A_{\mu}$, and $\mathcal{D}^{*}$ is its complex conjugate. Therefore,

$$
T_{\mu}^{\mu}=\frac{i}{2} c\left(\bar{\psi} \gamma^{\mu} \mathcal{D}_{\mu} \psi-\mathcal{D}_{\mu}^{*} \bar{\psi} \gamma^{\mu} \psi\right)=-m c^{2} \bar{\psi} \psi
$$

and, according to Eq. (13), the energy of a composite or elementary particle is

$$
E=m c^{2} \int \bar{\psi} \psi d V
$$

This equation is related to Fock's old result for the Dirac equation in an external, central Coulomb field [23] and also to the more general virial theorem of Rose and Welton [24] (see Ref. [11] as well).

Since we are considering the Dirac field $\psi$ as a classical field, constituting a sort of matter fluid, $n(\boldsymbol{x})=\bar{\psi} \psi$ is the total particle number density, with equal weight for particles and antiparticles, and computed in the local reference frame. Equation (17) might seem to imply that the bound-state energy is just the number of particles times the rest energy per particle, as if they were free and at rest, but it does not, because $n(\boldsymbol{x})$ must be computed in the local reference frame. When $n(\boldsymbol{x})$ is referred to the laboratory frame, namely, the bound-state rest frame, then

$$
m n(\boldsymbol{x}) \rightarrow r(\boldsymbol{x}) \sqrt{1-v(\boldsymbol{x})^{2} / c^{2}}
$$

where $r(\boldsymbol{x})$ is the ordinary non-relativistic mass density and $v(\boldsymbol{x})$ is the velocity of the matter-fluid element $d m=r(\boldsymbol{x}) d V$. Thus, we obtain

$$
E=c^{2} \int \sqrt{1-\frac{v(\boldsymbol{x})^{2}}{c^{2}}} r(\boldsymbol{x}) d V
$$

which is a continuous form of Eq. (5). Naturally, this virial theorem applies only to bound-state solutions of the nonlinear equations of the classical electrodynamics of the Dirac field, which are hardly explored (see, for example, Ref. [25]). As these equations have stable bound-state solutions, the corresponding single particle models do not need extraneous Poincaré stresses.

\subsection{Hamiltonian field theory formulation}

We can obtain a general field-theory virial theorem within the Hamiltonian formalism by generalizing the derivation of the virial theorem for a finite number of degrees of freedom in Sect. 2. Let us consider a generic field, which we denote $\varphi$ but which can comprise a set of independent fields (it can be a vector field, etc), and consider its Lagrangian and Hamiltonian densities, respectively, $\mathcal{L}$ and $\mathcal{H}$. The associated field momentum density is

$$
\pi=\frac{\partial \mathcal{L}}{\partial \dot{\varphi}} .
$$


In particle mechanics, dilatations simply act on the coordinates as $q \rightarrow l q$ and on the momenta as $p \rightarrow p / l$, which in infinitesimal form are given by Eq. (1). In field theory, dilatations primarily act on space coordinates and, through them, on field coordinates $\varphi$ and momenta $\pi$. Therefore, the infinitesimal generator of the finite dilatation $\varphi(\boldsymbol{x}) \rightarrow$ $l^{D} \varphi(l \boldsymbol{x})$, where $D$ is the dimension matrix, is given by the Lie derivative

$$
\delta \varphi=D \varphi-x^{i} \partial_{i} \varphi
$$

The dimension matrix $D$ can be assumed to be diagonal, that is to say, $\varphi$ can be assumed to be formed by eigenstates of $D$. The Lie derivative $\delta \varphi$ differs from $\delta q$ in Eq. (1) in the presence of the transport term, with $x^{i} \partial_{i}$, and also in the presence of $D$, which generalizes the trivial dimension of $q$. The canonical generator of dilatations $F$ such that $\delta \varphi=\delta F / \delta \pi$ is

$$
F=\int \pi\left(D \varphi-x^{i} \partial_{i} \varphi\right) d V
$$

Therefore,

$$
\delta \pi=-\frac{\delta F}{\delta \varphi}=(-3-D) \pi-x^{i} \partial_{i} \pi
$$

up to the vanishing of a surface integral. We deduce that both $\delta \varphi$ and $\delta \pi$ constitute the infinitesimal generators of Hamiltonian dilatations. Note that, under a finite dilatation, $\pi(\boldsymbol{x}) \varphi(\boldsymbol{x}) \rightarrow l^{-3} \pi(l \boldsymbol{x}) \varphi(l \boldsymbol{x})$, so that $F$ is invariant.

The generating function $F$ given by Eq. (19) is the integral of the sum of two parts, one corresponding to the intrinsic change of the field and another corresponding to the spatial transport. The latter can be written as $x^{i} T_{\mathrm{C}}{ }^{0}{ }_{i}$, where $T_{\mathrm{C}}{ }^{\mu}{ }_{\nu}$ comprises the four conserved currents associated to space-time translations by Noether's theorem [26]; that is to say, it is the canonical energy-momentum tensor. For example, for a scalar field $\varphi$ with $\mathcal{L}=-\partial^{\mu} \varphi \partial_{\mu} \varphi / 2-\mathcal{V}(\varphi)$

$$
T_{\mathrm{C}}{ }^{\mu}{ }_{\nu}=\partial^{\mu} \varphi \partial_{\nu} \varphi+\delta_{\nu}^{\mu} \mathcal{L}
$$

So

$$
T_{\mathrm{C}}{ }^{0}{ }_{i}=-\dot{\varphi} \partial_{i} \varphi=-\pi \partial_{i} \varphi
$$

For the electromagnetic field,

$$
T_{\mathrm{C}}{ }^{\mu}{ }_{\nu}=F^{\mu \rho} \partial_{\nu} A_{\rho}+\delta_{\nu}^{\mu} \mathcal{L}
$$

so, in the Hamiltonian gauge $A_{0}=0$,

$$
T_{\mathrm{C}}{ }^{0}{ }_{i}=-\dot{A}_{j} \partial_{i} A^{j}=-\pi_{j} \partial_{i} A^{j} .
$$

Therefore, in general,

$$
F=\int\left(\pi D \varphi+x^{i} T_{\mathrm{C}}{ }^{0}{ }_{i}\right) d V .
$$

However, $T_{\mathrm{C}}{ }^{0}{ }_{i}$ can be redefined by adding to it $\partial_{j} t_{i}^{j}$, where $t_{i}^{j}$ is an arbitrary function of $\varphi$ and $\pi$. With the appropriate choice of $t_{i}^{j}$, one can cancel the first summand in the 
right-hand side of Eq. (20) (up to a surface integral). In other words, there is always an "improvement" of the energy-momentum tensor such that the dilatation generator becomes

$$
F=\int x^{i} T_{i}^{0} d V
$$

This connects the Hamiltonian formulation of the virial theorem with Landau and Lifshitz's proof [9]. Indeed, using the conservation law $\partial_{\mu} T^{\mu \nu}=0$,

$$
\dot{F}=\int T^{i}{ }_{i} d V
$$

The vanishing of the temporal average of $\dot{F}$ and, therefore, of the spatial integral of the temporal average of $T^{i}{ }_{i}$ give rise to the virial theorem, Eq. (9).

Notice that one can also consider, instead of dilatations, general (anisotropic) coordinate transformations and so derive the tensor virial theorem.

The general condition of exact scale invariance is $\delta_{F} H=-\dot{F}=0$, or

$$
\dot{F}=\int T_{i}^{i} d V=0
$$

without averaging. This condition is not fulfilled by general field configurations of normal field theories. Naturally, $\dot{F}$ must vanish for any static field configuration and hence so does the integral of the stress tensor trace. This is in accord with Laue's theorem (Sec. 2.1), applicable to any static relativistic system and, in particular, to any model of an elementary particle, such as the electron. For example, for Bialynicki-Birula's electron model, the condition (21) is indeed fundamental for relativistic invariance [22].

Let us remark on one interesting consequence of Eq. (21) for static field configurations. In the case of a scalar field with $\mathcal{L}=-\partial^{\mu} \varphi \partial_{\mu} \varphi / 2-\mathcal{V}(\varphi)$, a static field has

$$
T_{\mathrm{C}}{ }^{i}{ }_{i}=\frac{2-d}{2}(\nabla \varphi)^{2}-d \mathcal{V}(\varphi)
$$

where $d$ is the space dimension (we simply use the canonical, unimproved energy-momentum tensor, because the space integral of the stress tensor is not altered by the improvement). With full generality, we can take $\mathcal{V}(\varphi) \geq 0$ and vanishing at its absolute minima; namely, we assume that there are several absolute minima with $\mathcal{V}=0$. Then, Eqs. (21) and (22) imply, for $d \geq 2$, that $\varphi(\boldsymbol{x})$ is constant and equal to its value at one of the minima. The absence of localized static solutions of scalar field theories in $d \geq 2$ is known as the HobartDerrick theorem $[27,28]$ and is usually proved by direct scaling of $\varphi(\boldsymbol{x})$. The generalization of this theorem to other field theories more complicated than the scalar field theory is also given by Eq. (21), although it can be proved by direct scaling of the appropriate field(s), case by case $[29$, Ch 6$]$.

In the current treatment of scale transformations, we have chosen examples of relativistic fields, but let us notice that there is no need to impose Lorentz invariance to obtain 
the virial theorem. When there is Lorentz invariance, the Lagrangian formulation of scale transformations, in terms of $\mathcal{L}$, is usually employed instead of the Hamiltonian formulation, because it is covariant and, hence, explicitly relativistic. The Lagrangian formulation of scale transformations is based on space-time dilatations, such that

$$
\delta \varphi=D \varphi-x^{\alpha} \partial_{\alpha} \varphi .
$$

They coincide with space-only dilatations for static fields. The local current associated to space-time dilatations by Noether's theorem can always be expressed as $j_{\mathrm{D}}^{\mu}=x_{\nu} T^{\mu \nu}$ and scale invariance can be expressed in the local form $\partial_{\mu} j_{\mathrm{D}}^{\mu}=0[29,30]$. Therefore, in field theory, scale invariance is generally connected with the tracelessness of the energy-momentum tensor. Although the conserved symmetric energy-momentum tensor of a scale-invariant field theory is not necessarily traceless, it is always possible to "improve" it and convert it into one that is traceless, in addition to symmetric and conserved [29, 30]. Then, the tracelessness of the energy-momentum tensor implies, beyond Poincaré and scale invariance, full invariance under the conformal group, which is obtained by adjoining the discrete inversion to Poincaré and scale transformations. The sourceless Maxwell equations are, of course, conformal invariant and, in this case, the energy-momentum tensor that results from symmetrizing its canonical form is already traceless [9], so it needs no improvement. However, such improvement is necessary in other field theories, e.g., in the massless scalar field theory.

\subsection{Scale invariance in the ultrarelativistic domain}

Regarding the virial theorem for an $N$-particle bound state in electrodynamics, Eq. (5), there arises the possibility of bound states of vanishing energy, namely, states such that $E \ll \sum_{a} m_{a} c^{2}$, as the bound particles become ultrarelativistic and approach the speed of light. In one such bound state, the kinetic energy tends to infinity, but this is compensated by a potential energy that tends to minus infinity, as the particles approach one another and the system collapses.

The vanishing of the energy of an ultrarelativistic bound state is a consequence of scale invariance. As remarked in page 6 , the relativistic form of mechanical similarity involves the scaling of mass, since mass is inextricably linked to energy in relativistic mechanics. Hence, full similarity demands the absence of masses. In the ultrarelativistic limit, $p_{a} \gg m_{a} c$, the electrodynamics Hamiltonian (3) becomes

$$
H=\sum_{a}\left|c \boldsymbol{p}_{a}-e \boldsymbol{A}\right|+e \Phi
$$

which is also obtained just by setting $m_{a}=0$. The absence of masses suggests scale invariance. Indeed, provided that $\boldsymbol{A}$ and $\Phi$ are homogeneous functions of degree $n=-1$, $H$ transforms into $H / l$ under the phase-space coordinate scaling $\boldsymbol{x} \rightarrow l \boldsymbol{x}$ and $\boldsymbol{p} \rightarrow \boldsymbol{p} / l$. In consequence, a system of ultrarelativistic particles is such that the energy is proportional to $p$ and, therefore, in the limit $p \rightarrow \infty$, a bound state must have $E=0$ exactly and be 
scale invariant. Otherwise, $E$ is a non-vanishing function of the $m_{a}$ and the bound state is not scale invariant.

One scale-invariant relativistic state of vanishing energy is the vacuum, which is neutral and has no measurable property. In fact, one can imagine that a neutral $N$-particle system loses energy by radiation and traverses a sequence of ultrarelativistic bound states of decreasing energy that ends in the vacuum. However, the study of the last stages of this process demands a quantum field theory treatment. As an example of decay to vacuum, one can consider the annihilation of positronium, but positronium is a weakly coupled system, such that its annihilation takes place before it enters the ultrarelativistic domain. Besides, one can also consider ultrarelativistic states with non-zero charge. A simple example of ultrarelativistic dynamics in atomic physics is briefly studied below. This example is useful, in particular, to introduce the phenomenon of vacuum decay, important in QCD (Sec. 3.2).

Although, apparently, there are no electromagnetically bound particles in ordinary matter that are fully relativistic, the fastest electrons of certain atoms actually are ultrarelativistic. Let us focus on one of the innermost and fastest electrons of a heavy atom, and, for simplicity, consider it as if it were not influenced by the other electrons; namely, let us consider the one-electron Hamiltonian

$$
H=c \sqrt{\boldsymbol{p}^{2}+(m c)^{2}}-\frac{Z e^{2}}{4 \pi r}
$$

(the nucleus can be taken at rest and, hence, can be neglected). For a circular orbit, the radial equation of motion just states the equality of centrifugal and Coulomb forces, which can be written in terms of the respective virials as

$$
\frac{c \boldsymbol{p}^{2}}{\sqrt{\boldsymbol{p}^{2}+(m c)^{2}}}=\frac{Z e^{2}}{4 \pi r} .
$$

For a general orbit, the equality of virials holds only on a temporal average. Given that

$$
\frac{c \boldsymbol{p}^{2}}{\sqrt{\boldsymbol{p}^{2}+(m c)^{2}}}=\boldsymbol{p} \cdot \boldsymbol{v}=\frac{m \boldsymbol{v}^{2}}{\sqrt{1-\boldsymbol{v}^{2} / c^{2}}},
$$

the temporal average of Eq. (24) is a particularly simple case of the virial theorem constituted by Eqs. (9), (10), (11), and (12), with $\boldsymbol{E}_{\mathrm{T}}=\boldsymbol{B}=0$. Also, note the connection with Eq. (6). As regards the energy, the virial theorem says that the ratio $E /\left(m c^{2}\right)$ equals the temporal average of $\sqrt{1-\boldsymbol{v}^{2} / c^{2}}=m c / \sqrt{\boldsymbol{p}^{2}+(m c)^{2}}$.

As usual, virial relations can be used to draw conclusions on the dynamics without solving the equations of motion. In particular, from Eq. (24) and

$$
\frac{1}{\sqrt{\boldsymbol{p}^{2}+(m c)^{2}}} \leq \frac{1}{p}
$$

we deduce that $c p \geq Z e^{2} /(4 \pi r)$, on the average. The equality $c p=Z e^{2} /(4 \pi r)$ takes place in the ultrarelativistic limit, in which $r \rightarrow 0, p \rightarrow \infty, E \rightarrow 0$, and scale invariance 
is approached. For a circular orbit, the angular momentum is $M=p r$, so we have the condition that $M \geq Z e^{2} /(4 \pi c)$. Actually, when $M<Z e^{2} /(4 \pi c)$, no orbit is stable and the electron must fall in towards the nucleus, in a spiral trajectory [9, §39]. In particular, as the electron approaches the nucleus and becomes ultrarelativistic, its trajectory tends to a logarithmic spiral, which is self-similar. Nevertheless, there are stable orbits for every $E>0$, although, as $E \rightarrow 0$, the only stable orbits are the circular orbits and they become just marginally stable. For $E<0$, there are no stable orbits.

Let us now consider the one-electron heavy atom in quantum mechanics, where the uncertainty principle sets a lower limit to the atom's size; namely, both $r$ and $E$ are bounded below and their lowest values correspond to the ground state of the Hamiltonian. This happens whether the electron is relativistic or not, but in the relativistic case, namely, for the $H$ of Eq. (23), a new feature arises: low $M$ states and hence states with the lowest positive energies can be unstable; that is to say, states with $M \sim \hbar$ are stable only if $Z \alpha<1$, where $\alpha=e^{2} /(4 \pi \hbar c)=1 / 137$. This is confirmed by solving the problem with a relativistic wave equation, for example, the Klein-Gordon, Dirac or Salpeter equations: the ground state is unstable for large $Z \alpha$, and the stability bound to $Z \alpha$, which depends on the wave equation considered, is always of the order of unity. ${ }^{2}$ This instability can be interpreted as a quantum mechanical collapse in which the standard vacuum decays and gives rise to a new, negatively charged vacuum $[32, \S 7]$. The charged vacuum can be pictured as an electron cloud attached to the nucleus. This cloud corresponds to the classical spiral trajectories that fall into the nucleus and is also asymptotically self-similar.

Let us remark that this vacuum instability occurs due to the presence of a nonelectromagnetic interaction, namely, the strong interaction, which keeps the positive charge $Z e$ within a nucleus sufficiently small as to produce a very strong electromagnetic field. In contrast, the electron-positron system does not have negative energy states, owing to the smallness of $\alpha$. A proper study of this problem demands quantum field theory methods, but the problem can be reduced, in a certain approximation, to an equation similar to the Schrödinger equation for the $H$ defined by Eq. (23) [33]. If there were electronpositron negative energy states, the standard QED vacuum would not be stable against condensation of electron-positron pairs. This vacuum decay does not occur in QED, but quark-antiquark condensation and vacuum decay take place in QCD [33]. This property of the QCD vacuum is crucial for hadron physics, as studied in Sec. 3.2.

Regarding scale invariance, the fundamental effect of quantum mechanics is to introduce the new constant $\hbar$, which together with $c$ leaves only one physical dimension, say, length. Therefore, particle masses can be associated with length scales, namely, their associated Compton wavelengths. Let us remark that this association is consistent with the Andersen-Baeyer [4] relativistic similarity. The Compton wavelength $\hbar /(m c)$ marks the scale where the momentum or energy uncertainties are large enough to allow the creation of a particle-antiparticle pair, so the very concept of particle loses meaning. The rele-

\footnotetext{
${ }^{2}$ The spectrum of the $H$ of Eq. (23) has been studied by Herbst [31]. He proves that Eq. (24) holds as an equation for the expectation values in eigenstates of $H$ and proves that the spectrum is non-negative for $Z \alpha \leq 2 / \pi$, whereas it is unbounded below for $Z \alpha>2 / \pi$.
} 
vant wavelength for an atom, namely, $\hbar /(m v) \sim \hbar /(m c Z \alpha)$ is definitely larger than the electron's Compton wavelength if $Z \alpha \ll 1$. In the opposite case, $Z \alpha \gtrsim 1$, the electron becomes ultrarelativistic and the potential of the (point-like) nucleus is strong enough to induce the creation of electron-positron pairs. As scale invariance can only take place in the ultrarelativistic domain, it takes place for length scales much smaller than $\hbar /(m c Z \alpha)$, where the one-electron description is inadequate. In general, any mass scale breaks scale invariance in relativistic quantum mechanics, and scale invariance can only be recovered in the ultrarelativistic domain, but in this domain the quantum effects associated with particle creation take over. It turns out that, in addition to the explicit breaking of scale invariance by any mass, scale invariance is always broken by quantum effects on small scales, even in massless systems $[29,30]$. The failure of a classical symmetry due to quantum effects is called a quantum anomaly. The scale invariance anomaly is important for bound states, especially in QCD, and features in the formulation of a QFT virial theorem (see next section).

\section{The quantum field theory virial theorem}

In relativistic quantum mechanics, scale invariance is broken on scales of the order of the particles' Compton wavelengths, as said above. On the other hand, on these scales, a bound system cannot be described in terms of a definite set of particles that interact through a field, because the Schrödinger equation for that set of particles neglects the possible creation of more particles. As is well known, relativistic quantum mechanics leads to quantum field theory, in which particles and fields are on the same footing, so the Schrödinger equation is best expressed in terms of all the fields present. Therefore, the virial theorem for a definite set of elementary particles given by Eq. (5) is naturally replaced by the field theory formulations given by Eq. (9) or Eq. (13). The quantum versions of these forms of the virial theorem can be derived by analogy with the Landau-Lifshitz proof [12] or directly from Eq. (21), yielding:

$$
\int\left\langle T_{i}^{i}\right\rangle d V=0, \quad E=-\int\left\langle T_{\mu}^{\mu}\right\rangle d V,
$$

where the expectations values are taken with respect to a normalized stationary state representing a bound state in its rest frame.

If a semiclassical (perturbative) expansion is meaningful, Eqs. (25) amount to the classical field theory virial theorem, namely, Eq. (9) or Eq. (13), plus quantum corrections. The simplest quantum correction consists in employing the limited Fock space approximation [33], which is a variational approximation equivalent to the Schrödinger equation in a limited Fock space that does not include renormalization effects. When applied to an electron-positron bound state [12], the result is that its energy $E$ can be expressed by either Eq. (7), without $U$ terms and with $m_{1}=m_{2}$, of course, or by Eq. (18). In both equations, the classical motion is replaced with a probability distribution, given by a quantum wave-function for the former and by a classical "mass density" for the latter. 
The limited Fock space approximation for bound states is connected with the standard treatment of bound states in QFT, which involves several approximations that lead to the Bethe-Salpeter equation $[32, \S 6]$.

In the limited Fock space approximation for spinor QED no infinities arise [33, 12], but, in general, one must consider the infinities that inevitably arise in QFT. Those infinities require regularization with an ultraviolet (UV) cutoff, which necessarily breaks scale invariance. However, let us remark that some infinities already arise in a classical field theory with point-like particles and because of this problem the classical electrodynamics of particles of mass $m$ and charge $e$ is not consistent on scales smaller than the classical radius $e^{2} /\left(m c^{2}\right)[9,14]$. In fact, as explained in Sec. 2.1, the classical relativistic virial theorem holds only after subtraction of the infinite self-energy of point-like charges. Since the classical radius is smaller than the Compton wavelength $\hbar /(m c)$, where the quantum effects take over, the regularization of infinities is essentially a quantum problem. ${ }^{3}$ For the virial theorem in the form of Eq. (25), the relevant quantum effects of renormalization manifest themselves in the energy-momentum tensor trace anomaly, as pointed out by Dudas and Pirjol [12].

\subsection{The QED virial theorem and the trace anomaly}

Before considering the renormalization process for bound states in QFT, let us recall why renormalization is necessary for the classical virial theorem to hold (Sec. 2.1). For a set of electromagnetically bound particles, fulfilling Eq. (10), the virial theorem of Eq. (9) implies that their positive kinetic "pressure" must be balanced by negative electromagnetic stresses. Therefore, their electrostatic energy, as given by Eq. (12), must be negative, despite that it appears to be positive. In fact, this energy is actually infinite, but it can become negative after suitable subtractions, absorbed by mass renormalizations. Mass renormalization is also necessary in QFT because of the appearance of infinite self-energies, but the divergence structure is substantially modified. Besides, in QFT, the charge must be renormalized as well.

In a semiclassical expansion, the calculation of the first quantum correction to some bound state energy only requires the calculation of the small oscillations of the corresponding classical solution. For example, for a heavy atom, this semiclassical approach is equivalent to the old Bloch hydrodynamic treatment of the Thomas-Fermi atom model [34]. However, this model is non-relativistic. Proper relativistic examples are provided by classical relativistic field theory localized solutions ("classical lumps") [29, Ch 6]. In general, the calculation of quantum corrections begins with the calculation of the stability matrix determining the small oscillations. The total energy is the classical energy plus the contribution of these small oscillations. Once these oscillations are quantized, the first

\footnotetext{
${ }^{3}$ This statement must be qualified: In the exceptional case in which the strong interaction holds a charge $Z e$ in a point-like nucleus, $e^{2}$ has to be replaced with $Z e^{2}$, and the condition $Z \alpha>1$ precisely means that $Z e^{2} /\left(m c^{2}\right)$ is larger than $\hbar /(m c)$.
} 
quantum correction to the classical energy is

$$
\delta E=\frac{\hbar}{2} \sum_{i} \omega_{i}
$$

where the sum goes over the oscillation modes and $\omega_{i}$ is the angular frequency of the $i$-th mode. This sum diverges at high frequency (in the UV). The modes can be labeled by three independent numbers that can be assembled, for large values, in a wave-number vector $\boldsymbol{k}$. Therefore, the UV divergence can be segregated by expressing the sum as an integral over $\boldsymbol{k}$, for large $k$, and taking $\omega_{i} \approx c k$, as corresponds to free modes; namely,

$$
\delta E=\hbar c V \int^{\Lambda}[k+\mathrm{O}(1)] \frac{d^{3} k}{(2 \pi)^{3}}=\frac{\hbar c}{8 \pi^{2}} V\left[\Lambda^{4}+\mathrm{O}\left(\Lambda^{3}\right)\right]
$$

where $V$ is the system's volume, a UV cutoff $\Lambda$ has been introduced, and a sum over two polarizations has been made, assuming that the free modes correspond to photons. One could make $\delta E$ finite and attribute it a physical meaning by choosing a physical cutoff $\Lambda$, such as $\Lambda \sim m c / \hbar$, namely, the inverse of the electron Compton wavelength. At any rate, the leading term, which is positive and proportional to $\Lambda^{4}$, is present in the absence of matter and must be subtracted. The subleading divergent terms depend on the detailed spectrum $\omega_{i}$ and hence on the matter state, and they can have either sign. After renormalization (or definition of a finite $\Lambda$ ), these terms give rise to measurable electromagnetic energies.

The divergent parts of the vacuum energy indeed have to be subtracted in the calculations of electromagnetic energies, for example, in the calculation of Casimir or Van der Waals forces [35]. For illustration, let us briefly consider a case in which the frequencies $\omega_{i}$ are easy to calculate, namely, the case of a dilute gas of $N$ atoms per unit volume in a box of volume $V[35, \S 3.7]$. The allowed frequencies are the free-field frequencies modified by the refractive index of the gas, $n(\omega)$, i.e. $\omega_{i} \approx c k / n$. If we assume, for simplicity, that there is only one resonant frequency, $\omega_{0}$, we can take

$$
n(\omega)=1+\frac{N e^{2}}{2 m\left(\omega_{0}^{2}-\omega^{2}\right)} .
$$

Therefore,

$\delta E=\frac{\hbar c V}{(2 \pi)^{3}} \int \frac{k d^{3} k}{n(k)}=\frac{\hbar c V}{2 \pi^{2}}\left[\frac{\Lambda^{4}}{4}+\frac{N e^{2} \Lambda^{2}}{4 m c^{2}}+\frac{N e^{2}}{4 m c^{2}}\left(k_{0}^{2}+\frac{N e^{2}}{2 m c^{2}}\right) \ln \left|\frac{\Lambda^{2}}{k_{0}^{2}+N e^{2} /\left(2 m c^{2}\right)}-1\right|\right]$.

The quadratic divergence is proportional to the number of atoms, $N V$, but is independent of $\omega_{0}$, and the proportionality constant only depends on fundamental constants. Indeed, this term corresponds to the energy of $N V$ free electrons, and, if we take again $\Lambda \sim m c / \hbar$, the energy per electron is of the order of $e^{2} /(\hbar / m c)$, that is to say, of the electron quantum self-energy. Therefore, this divergence can be absorbed by mass renormalization. The logarithmic term is of smaller magnitude and depends on $\omega_{0}$. If $N e^{2} / m \ll \omega_{0}^{2}$ (the dilution 
condition), the logarithmic term can be identified with the Lamb shift [35, § 3.7]. Notice that the quantum corrections are, after renormalization, not only finite but small (of order $\alpha)$. If we take, instead of $\Lambda \sim m c / \hbar$, the much smaller cutoff $\Lambda=k_{0}$, so that $n(k)>1$ over the full range of integration, then $\delta E$ is negative, as corresponds to attractive Van der Waals forces.

The result of the preceding calculation of $\delta E$ and, in particular, its cutoff dependence are typical of one-loop effective potential calculations. Indeed, the energy $E$ can be generally calculated in terms of the minimum of the effective action corresponding to the bound state. The $\Lambda^{4}$ term is already present in the vacuum, when a generic regularization method is employed, and leads to the well-known cosmological constant problem. In this regard, Ossola and Sirlin [36] study the contribution of fundamental particles to the vacuum energy density, comparing various regularization methods, and conclude that, for non-interacting particles, the divergence can be made quadratic rather than quartic (no $\Lambda^{4}$ term), and that massless particles do not contribute. This result follows from properly considering relativistic covariance and scale invariance of free field theories in the massless limit and, therefore, calculating $\left\langle T^{00}\right\rangle$ or any other component of $\left\langle T^{\mu \nu}\right\rangle$ in terms of the trace $\left\langle T_{\lambda}^{\lambda}\right\rangle$, by employing the relation $\left\langle T^{\mu \nu}\right\rangle=\left\langle T_{\lambda}^{\lambda}\right\rangle g^{\mu \nu} / 4$. The divergences of the trace $\left\langle T_{\lambda}^{\lambda}\right\rangle$ are easily obtained for free fields, but, with interactions, new divergences appear, namely, new quartic, quadratic and logarithmic divergences. After renormalization, the energy-momentum tensor trace has quantum corrections, that is, a trace anomaly appears. In particular, the trace is not zero even in the massless case. As the virial theorem can be expressed in terms of the energy-momentum tensor trace, one concludes that the virial theorem must include a quantum trace anomaly term [12], since this anomaly appears even in the vacuum.

The general form of the fermionic QED trace anomaly was computed by Adler et al $[37]:$

$$
T_{\mu}^{\mu}=-K_{1} m_{0} c^{2} \bar{\psi} \psi+K_{2} N\left[F_{\lambda \sigma} F^{\lambda \sigma}\right]
$$

where $N$ indicates a type of normal product (see [37]) and

$$
\begin{array}{r}
K_{1}=1+\delta(\alpha)=1+\frac{3 \alpha}{2 \pi}+\cdots \\
K_{2}=\frac{1}{4} \beta(\alpha)=\frac{1}{4}\left(\frac{2 \alpha}{3 \pi}+\frac{\alpha^{2}}{2 \pi^{2}}+\cdots\right) .
\end{array}
$$

The functions $\delta$ and $\beta$ are associated to the anomalous dimension of the fermion field $\psi$ and to the coupling constant renormalization, respectively. Notice that the first term of the trace anomaly consists of the classical part in Eq. (16) plus quantum corrections due to mass renormalization, whereas the second term is purely of quantum origin and is due to charge renormalization.

The QED trace anomaly is indeed small, due to the smallness of $\alpha$, which makes perturbation theory work. This implies that the renormalized value of the quantum correction $\delta E$ to a classical bound-state energy is small as well. Fundamentally, it implies that the vacuum is not altered, that is to say, that there is no vacuum decay. Naturally, the vacuum decay in the strong field of a nucleus is an exception, due to the actual coupling 
constant, $Z \alpha$, not being small (Sec. 2.3). At any rate, it is interesting to consider general strongly-bound states. A state can be considered strongly bound if the largest part of its energy is due to the interaction of its constituents rather than to their rest masses. This condition is naturally met by hadronic states, namely, the states of quarks bound by the strong interaction described by QCD.

\subsection{Scale symmetry breaking, Callan-Symanzik equations, and QCD}

QCD is the theory of the strong interaction, although effective low-energy theories, such as the meson-nucleon interaction theory, are still useful. The meson-nucleon theory that only includes the lightest mesons is somewhat similar to the photon-electron theory in QED, except for the finite range of the mesons. However, the magnitude of the meson-nucleon coupling is $g^{2} /(4 \pi \hbar c) \simeq 1$, which should imply that the bound states are ultrarelativistic and their energies nearly vanish! This does not happen, of course, because scale invariance is badly broken by quantum effects. Coleman [29, Ch 3] employs the meson-nucleon model for illustrating the breaking of scale invariance, by analyzing the perturbative behavior of correlation functions in the deep Euclidean region: in addition to simple powers of the scale (given by the field dimensions), there appear logarithms as well. Then, Coleman shows that some series of logarithms can be absorbed into anomalous field dimensions, while other logarithms remain, but the series of the latter can be absorbed by a renormalization of the couplings. In general, the scale dependence introduced by the renormalization process can be expressed in terms of a set of simple differential equations for the correlation functions, namely, the renormalizations group equations, while the effect of scale-symmetry breaking is expressed by the Callan-Symanzik equations. Naturally, both sets of equations are related [29, Ch 3]. There is an infinite set of Callan-Symanzik equations, one equation for every correlation function. To apply these equations to bound states, it is useful to realize that they all can be derived from a master Callan-Symanzik equation, which is, in addition, connected with the energy-momentum tensor trace anomaly.

To obtain the master Callan-Symanzik equation, let us introduce the generating functional

$$
Z\left[\lambda^{j}, g_{\mu \nu}\right]=\int \mathcal{D} \varphi \exp \left(i S\left[\varphi, \lambda^{j}\right]\right)
$$

namely, the vacuum transition amplitude, where the action $S$ depends on a set of fields $\varphi$ and coupling constants $\lambda^{j}$ and is defined in a curved space-time, and let us also introduce the functional $W=-i \log Z$. The effect of a scale transformation can be alternately realized through the metric, so

$$
l \frac{d W}{d l}=2 \int d^{4} x g^{\mu \nu}(x) \frac{\delta W}{\delta g^{\mu \nu}(x)}=\int d^{4} x \sqrt{\operatorname{det} g_{\mu \nu}}\left\langle T_{\mu}^{\mu}\right\rangle,
$$

where $l$ is the scale, or through the coupling constants, so

$$
l \frac{d W}{d l}=\sum_{i} \beta^{i}(\lambda) \int d^{4} x \sqrt{\operatorname{det} g_{\mu \nu}}\left\langle\mathcal{O}_{i}\right\rangle+\mathcal{A},
$$


where $\beta^{i}(\lambda)=l d \lambda^{i} / d l$ ("beta functions"), $\left\{\mathcal{O}_{i}\right\}$ is the set of interaction terms (a subset of "composite fields"), fulfilling

$$
\frac{\delta W}{\delta \lambda^{i}}=\int d^{4} x \sqrt{\operatorname{det} g_{\mu \nu}}\left\langle\mathcal{O}_{i}\right\rangle,
$$

and $\mathcal{A}$ is the extra anomalous term that arises in a curved geometry. Therefore, in flat space-time,

$$
\int d^{4} x\left\langle T_{\mu}^{\mu}\right\rangle=\sum_{i} \beta^{i}(\lambda) \int d^{4} x\left\langle\mathcal{O}_{i}\right\rangle .
$$

This equation ${ }^{4}$ generates an infinite hierarchy of equations for the correlations of the $\mathcal{O}_{i}$ by taking derivatives of it with respect to the couplings $\lambda^{i}$. Equations for the correlations of fields other than $\mathcal{O}_{i}$ and, in particular, of the elementary fields $\varphi$ can also be obtained by introducing in $W$ the respective sources. These equations are in essence the standard Callan-Symanzik equations. The master Callan-Symanzik equation (27) shows the general form of the trace anomaly [40]. For example, in QED, Eq. (27) is equivalent to the operator equation (26). To see this, one has to notice that the set $\left\{\lambda^{i}\right\}$ of coupling constants includes dimensionful constants. However, the dimensionless coupling constants play a special role, because they can contribute to each $\beta^{i}(\lambda)$, whereas the dimensionful coupling constants (corresponding to super-renormalizable interactions, e.g., mass terms) can only contribute to some of them, as a simple power-counting argument shows. Indeed, in Eq. (26), $\alpha$ contributes to both terms but $m_{0}$ is only allowed in the first term and with exponent one. Also, notice that the "beta functions" of dimensionful coupling constants are classically non-vanishing and give rise to the classical value of $T_{\mu}^{\mu}$, whereas those of dimensionless coupling constants are purely "anomalous."

Equation (27) can be generalized by replacing the vacuum expectation values with the expectation values in the stationary state corresponding to a bound state, namely, by introducing the appropriate boundary conditions in the generating functional $Z$. For stationary states, the four-dimensional integrals in Eq. (27) become spatial integrals and the equation directly yields the quantum corrections to the relativistic virial theorem (13), in terms of the beta functions and the expectation values $\left\langle\mathcal{O}_{i}\right\rangle$. The beta functions can be calculated in perturbation theory, but the $\left\langle\mathcal{O}_{i}\right\rangle$ are harder to calculate, because they are essentially non-perturbative.

To appreciate the importance of quantum corrections to the relativistic virial theorem, let us consider the theory of hadrons in QCD. As is well known, the crucial difference between QED and QCD is that their beta functions have opposite signs, so that QCD is asymptotically free, namely, the interaction vanishes at high momenta. In contrast, quarks interact strongly at low momenta, and, in fact, hadrons made of light quarks have a size such that the contributions of the interactions to their energy are much larger than the contribution of the quark masses. At the same time, the contribution of the trace anomaly is crucial. To clarify all this, let us consider a concrete hadron model.

\footnotetext{
${ }^{4}$ This equation is well known. It plays an important role in $2 \mathrm{~d}$ field theory $[38,39]$ and it is discussed, in general, by Osborn [40].
} 
In the MIT bag model [41], a constant positive potential energy $B$, per unit volume, is added to a free-field Lagrangian density inside a finite part of space. A semiclassical description of this simple field theory constitutes a sort of statistical model of hadrons. In the MIT bag model, the particles, named then "partons" but now identified with quarks and gluons, are free and massless inside the bag and they move with the speed of light. Therefore, hadrons are truly ultrarelativistic bound states, as studied in Sec. 2.3. The vacuum inside the bag takes place for small distances and high momentum and hence corresponds to the QCD perturbative vacuum. As the particles inside the bag separate from one another, they enter the strong coupling phase, in which the perturbative vacuum is not stable and decays to the standard QCD vacuum (a condensate of quark-antiquark pairs). Vice versa, the QCD vacuum becomes unstable at high momenta and decays to the perturbative vacuum. The transition is assumed to take place over a very small distance, at the bag's surface. In the statistical model, the confining interaction is just modeled as a vacuum pressure, namely, the difference between the null vacuum pressure outside the bag and the negative "vacuum pressure" in the bag. The effect of this pressure difference is just to keep the particles inside the bag, as if the difference of pressures were produced by the bag surface.

The application of the virial theorem to the gas of free massless particles of the statistical bag model is straightforward: the theorem just states that the energy density is three times the pressure difference $[9, \S 35]$. On the other hand, the energy-momentum tensor of the perturbative vacuum is constant and proportional to the metric $g^{\mu \nu}$, as corresponds to a constant term in the Lagrangian. Therefore, we have the following relations: the virial relation $E_{\text {quarks+gluons }} / V=3 P_{\text {quarks+gluons }}$ (where $V$ denotes the bag's volume), the balance of pressure in the bag, $P_{\text {quarks+gluons }}=-P_{\text {vac }}$, and the vacuum relation $E_{\text {vac }}=-P_{\text {vac }} V$. Let us remark that the balance of pressure is also a virial relation, namely, a particular case of $\int \overline{T_{i}^{i}} d V=0$. All the preceding equations together imply that

$$
E_{\text {quarks+gluons }}=3 E_{\mathrm{vac}}, \quad E=E_{\text {quarks+gluons }}+E_{\mathrm{vac}}=4 E_{\mathrm{vac}}=4 B V \text {. }
$$

Notice that the confining interaction accounts for one fourth of the total energy, like in the classical model of a particle with electric charge of Sec. 2.1 that has a confining Poincaré energy-momentum tensor proportional to $g^{\mu \nu}$.

The bag surface has been taken to be fixed but this is not necessary: the only change when the bag surface is allowed to move is that $V$ must be replaced with the average $\bar{V}$, so $E=4 B \bar{V}$. This equation is also obtained by Chodos et al [41] as a relativistic virial theorem specially tailored for the bag model Lagrangian and proved without employing the energy-momentum tensor.

Since the (improved) classical energy-momentum tensor is traceless in the MIT bag model, confinement is due to the quantum energy-momentum tensor trace part (the part proportional to $g^{\mu \nu}$ ), namely, to the the trace anomaly. In the MIT bag model, this quantity is spatially constant, but in QCD the trace anomaly is given by an equation similar to Eq. (26) that includes suitable color indices; namely, it contains the term $\bar{\psi} m \psi$, where $m$ is the quark mass matrix, and the term $F^{2}$, with the color indices contracted. According 
to the virial theorem (25) and assuming that the quarks are massless, the energy is given by the spatial integral of $-\left\langle T_{\lambda}^{\lambda}\right\rangle=-\beta(g)\left\langle F^{2}\right\rangle /(2 g)$ (in the remainder, we take $\hbar=c=1$ ). Therefore, at first order in $\alpha_{\mathrm{s}}=g^{2} /(4 \pi)$,

$$
E=-\int d V\left\langle T_{\lambda}^{\lambda}\right\rangle=\frac{9 \alpha_{\mathrm{s}}}{8 \pi} \int d V\left\langle F^{2}\right\rangle
$$

(for three quark flavors). The last equation is a particular case of Eq. (27), for a stationary state and only one coupling. The required spatial integral,

$$
\int\left\langle F^{2}\right\rangle d V=-2 \int\left(\boldsymbol{E}^{2}-\boldsymbol{B}^{2}\right) d V
$$

must be calculated with the bag boundary conditions. For the lowest energy states, the integral can be calculated in terms of the respective integrals of $\boldsymbol{E}^{2}$ and $\boldsymbol{B}^{2}$, which also appear in the calculation of the gluonic corrections to the ground states of the MIT bag model $[42, \S 3.12]$. These integrals contain divergent terms that correspond to self-interactions that are absorbed by mass renormalizations. The final result is that the electric integral vanishes and the magnetic integral is proportional to $1 / R$, namely, to the inverse of the spherical bag radius, with a coefficient of proportionality of the order of unity, as one should expect. Indeed, the partons in the lowest excitation states of the bag must have energy of the order of $N V^{-1 / 3}$, where $N$ is a small integer, as follows from dimensional analysis [41]. The trace anomaly calculation cannot specify $N$, since $\alpha_{\mathrm{s}}$ is undetermined.

Taking any one of the lowest energy hadronic states, the bag relation $E=4 B V \sim N / R$, with $V \sim R^{3}$, implies that $B \sim R^{-4}$, where $R$ is the hadron radius. Therefore, the fundamental dimensional parameter $B$ can be traded for $R$. However, let us introduce instead a fundamental QCD dimensional parameter, namely, the RG invariant $\Lambda_{\mathrm{QCD}}$, which naturally arises in massless QCD through the renormalization process, constituting what is called dimensional transmutation. $\Lambda_{\mathrm{QCD}}$ can be defined, for example, as the scale at which the running QCD coupling equals unity, $\alpha_{\mathrm{s}}\left(\Lambda_{\mathrm{QCD}}\right)=1$. The scale $\Lambda_{\mathrm{QCD}}$ determines the size of the lowest excitation states of hadrons, namely, $R \sim 1 / \Lambda_{\mathrm{QCD}}$. Therefore, we have $B \sim \Lambda_{\mathrm{QCD}}^{4}$ and $E_{\mathrm{vac}}=B V \sim \Lambda_{\mathrm{QCD}}$. Also, $E \sim \Lambda_{\mathrm{QCD}}$. Naturally, $R$ and $E$ can be expressed in terms of $\Lambda_{\mathrm{QCD}}$ for general hadron models with massless quarks, not only for the MIT bag model, as deduced from dimensional transmutation. Dimensional transmutation is a consequence of the breaking of scale invariance, which also gives rise to the trace anomaly. However, let us remark that the virial theorem only allows us to establish one relation, which is exact and says, in essence, that the confining term, namely, the trace anomaly, accounts for one fourth of the total energy, for massless quarks, as in Eq. (28). This exact relation derives from the obvious equation $T^{\mu \nu}=T_{\text {quarks+gluons }}^{\mu \nu}+T_{\alpha}^{\alpha} g^{\mu \nu} / 4$, where, classically, $T_{\alpha}^{\alpha}=0$, and

$$
T_{\text {quarks+gluons }}^{\mu \nu}=\frac{i}{2}\left(\bar{\psi} \gamma^{(\mu} \mathcal{D}^{\nu)} \psi-\mathcal{D}^{*(\mu} \bar{\psi} \gamma^{\nu)} \psi\right)-\frac{g^{\mu \nu}}{4} F^{2}+F^{\mu \sigma} \cdot F_{\sigma}^{\nu}
$$

Therefore, on account of the QFT virial theorem, Eq. (25),

$$
E=\int\left\langle T_{\text {quarks+gluons }}^{00}\right\rangle d V+\frac{E}{4}
$$


in analogy with Eq. (14). Naturally, $T_{\alpha}^{\alpha}=0$ implies $E=0$, but the trace anomaly makes $E$ non-vanishing. Equation (29) is exact for massless QCD and is approximately valid for hadrons made of light quarks. On the other hand, there is another relation, always approximate, that determines the size of the lowest excitation states of hadrons in terms of a fundamental parameter, say, $\Lambda_{\mathrm{QCD}}$ (or $B$, in the bag model). Obviously, the latter relation cannot hold for high excitation states, whereas the virial theorem does.

In more elaborate hadron models, one can consider quark masses, $m_{\mathrm{Q}}$, so that the classical energy-momentum tensor is not traceless. Nevertheless, for light quarks, namely, the $u$ and $d$ quarks (and surely the $s$ quark as well), $m_{\mathrm{Q}} \ll \Lambda_{\mathrm{QCD}}$, so the trace anomaly is still dominated by the $F^{2}$ term. If Nature were such that $\Lambda_{\mathrm{QCD}} \ll m_{\mathrm{Q}}$ for all quarks, then QCD, in spite of still having the property of quark confinement and still being asymptotically free, would share some features of QED: all hadrons would actually be non-relativistic quark bound states.

\section{Conclusions}

The virial theorem expresses the condition of average equilibrium of a bound state, namely, the condition that its average shape stays constant and, in particular, that its average size stays constant. The former condition is expressed as the tensor virial theorem, whereas the latter is expressed as the ordinary scalar virial theorem, Eq. (9), which is actually the trace of the tensor virial theorem. This theorem implies that the positive pressure of particles or fields corresponding to a traceless energy-momentum tensor must be compensated for by the negative stresses corresponding to the trace part of the energy-momentum tensor. Eq. (9) is valid in both classical and relativistic physics, but in the latter the equivalent form in terms of the energy-momentum tensor trace, Eq. (13), is more convenient. This equation implies that the proportion of the bound state energy corresponding to a traceless energy-momentum tensor is three fourths. This fraction is connected, in particular, with the $4 / 3$ that appears in the classic problem of electromagnetic inertia.

Both the trace of the stress tensor and the trace of the energy-momentum tensor are related to the generators of, respectively, space and space-time dilatations. Naturally, the connection with dilatations arises because these are the transformations that change the size of the system. The virial theorem shows that the average size of the system is determined by its energy $E$. Full space-time scale invariance only takes place when the (average of the) trace of the energy-momentum tensor vanishes, which corresponds to $E=0$ and, in principle, to the vacuum.

While non-relativistic mechanics admits similarity of motion for kinetic and potential energies that are homogeneous functions of their respective variables, this symmetry is lost in relativistic mechanics, in which the kinetic energy is never a homogeneous function. However, there exists a relativistic notion of similarity, which is such that velocities are left invariant but masses scale as energies do. Therefore, the only situation in which full scale invariance can appear is in the ultrarelativistic domain, when the masses are set to zero and the virial theorem implies that the energy vanishes. This ultrarelativistic scale 
invariance appears, for example, in the strong electric field of a heavy nucleus.

On the other hand, only in the context of quantum field theory does scale invariance acquire its deepest meaning, since there is naturally only one scale, either length or mass. In a theory with massless fundamental particles, bound systems should have $E=0$ and be massless as well, so there should be no scales and the theory would naturally be scale invariant. However, in quantum field theory the vacuum is non trivial, and there arise virtual particle-antiparticle pairs that make quantum contributions to energies that are actually infinite and, after renormalization, bring about a scale dependence, expressed by the Callan-Symanzik (or renormalization group) equations. This dilatation symmetry breaking can also be expressed in terms of the energy-momentum trace anomaly, which must be included in the quantum field theory virial theorem. Therefore, this theorem is just a generalization of the Callan-Symanzik or trace anomaly equations that is applicable to bound states. Full scale invariance takes place only at renormalization group fixed points, where the virial theorem becomes trivial.

The QED energy-momentum tensor trace anomaly is proportional to (powers of) the coupling constant $\alpha=1 / 137$, so it is small. In general, the quantum corrections to relativistic bound states are small in QED. As those bound states are weakly coupled, they are weakly relativistic as well. Exceptionally, the strong nuclear interaction within a heavy nucleus creates a concentration of positive charges such that their electromagnetic field is sufficiently strong to produce important relativistic effects, in particular, a qualitative change in the quantum vacuum around the nucleus: an electron bound to the nucleus can have negative energy, and the bound state actually decays to a new vacuum state with zero energy.

If the QED coupling $\alpha$ were sufficiently large, then vacuum instability would not be exceptional, because then positronium could have a negative energy state, and the standard QED vacuum would be unstable against production of electron-positron pairs. A phenomenon of this kind takes place in QCD, so the QCD vacuum is not the perturbative vacuum but a condensate of quark-antiquark pairs. This phenomenon is associated with the magnitudes of dilatation symmetry breaking and of the trace anomaly in QCD. In fact, the existence of hadrons in massless QCD is conditioned by the trace anomaly, which is the essential ingredient of the virial theorem in this case. The dilatation symmetry breaking can be expressed as a dimensional transmutation, which gives rise to a scale, the renormalization group invariant $\Lambda_{\mathrm{QCD}}$. This scale is definitely larger than the light quark masses, which implies that the essential properties of the hadrons formed by them are described by massless QCD. Assuming that quarks are massless, the virial theorem implies that one fourth of the energy (or mass) of a hadron comes from the trace anomaly term whereas three fourths come from quarks and gluons. The bag model of hadrons is useful for a straightforward application of the virial theorem and the computation of the trace anomaly, as has been shown.

A different type of strong interaction occurs in astrophysics, namely, in compact bodies, where the additive nature of gravity leads to strong gravitational fields, besides strong quantum effects. However, the generalization of the virial theorem to General Relativity presents problems that are beyond the scope of this paper, as cautioned in the introduc- 
tion. Nevertheless, we can affirm that the master Callan-Symanzik equation (27) that includes the curvature dependent trace anomaly $\mathcal{A}$ must play an important role in such a generalization.

\section{Acknowledgments}

I thank Sergey Apenko and Andrey Semenov for comments on the manuscript.

\section{References}

[1] Collins G W 1978 The Virial Theorem in Stellar Astrophysics (Tucson, AZ: Pachart)

[2] Landau L and Lifshitz E 1976 Mechanics 3rd edn (Oxford: Pergamon)

[3] Löwdin P O 1959 J. Mol. Spectrosc. 3 46-66

[4] Andersen C M and von Baeyer H C 1971 Am. J. Phys. 39 914-9

[5] Kleban P 1979 Am. J. Phys. 47 883-6

[6] van Kampen N G 1972 Rep. Math. Phys. 3 235-9

[7] Nachtergaele B and Verbeure A 1986 J. Geom. Phys. 3 315-25

[8] Bludman S and Kennedy D C 2011 J. Math. Phys. 52042902

[9] Landau L and Lifshitz E 1962 The Classical Theory of Fields 2nd edn (Oxford: Pergamon)

[10] Rafelsky J 1977 Phys. Rev. D 161890

[11] Brack M 1983 Phys. Rev. D 271950

[12] Dudas E A and Pirjol D 1991 Physics Letters B 260186

[13] Goldstein H 1950 Classical Mechanics (Cambridge Mass: Addison-Wesley) p 214

[14] Jackson J D 1999 Classical Electrodynamics 3rd edition (John Wiley)

[15] Anderson J L 1967 Principles of Relativity Physics (NY: Academic Press)

[16] Barut A O 1980 Electrodynamics and Classical Theory of Fields and Particles (NY: Dover)

[17] Lucha W and Schöberl F F 1990 Phys. Rev. Lett. 642733

[18] Hwang D S, Kim C S and Namgung W 1997 Phys. Lett. B 406117 
[19] von Laue M 1911 Annalen Phys. 35524

[20] Ohanian H C 2009 Studies in History and Philosophy of Modern Physics 40 167-173

[21] Hawking S W and Ellis G F R 1973 The large scale structure of space-time (Cambridge U. P.)

[22] Bialynicki-Birula I 1983 Phys. Rev. D 282114

[23] Fock V 1930 Zeitschrift für Physik 63 855-858

[24] Rose M E and Welton T A 1952 Phys. Rev. 86 432-433

[25] Wakano M 1966 Prog. Theor. Phys. 35 1117-1141

Radford C 2003 J. Phys. A 36 5663-5681

[26] Weinberg S 1995 The Quantum Theory of Fields vol I (Cambridge U. P.)

[27] Hobart R H 1963 Proc. Phys. Soc. 82 201-203

[28] Derrick G H 1964 J. Math. Phys. 5 1252-1254

[29] Coleman S 1985 Aspects of Symmetry (Cambridge U. P.)

[30] Callan C G Jr, Coleman S and Jackiw R 1970 Annals of Physics 59 42-73

[31] Herbst I W 1977 Commun. Math. Phys. 53 285-294; Commun. Math. Phys. 55316 (addendum)

[32] Greiner W and Reinhardt J 2003 Quantum Electrodynamics (Berlin: Springer-Verlag)

[33] Finger J, Horn D and Mandula J E 1979 Phys. Rev. D 123253

[34] Ball J A, Wheeler J A and Firemen E L 1973 Rev. Mod. Phys. 45333

[35] Milonni P W 1994 The Quantum Vacuum: an introduction to Quantum Electrodynamics (San Diego, CA: Academic Press)

[36] Ossola G and Sirlin A 2003 Eur. Phys. J. C 31165

[37] Adler S L, Collins J C and Duncan A 1977 Phys. Rev. D 151712

[38] Zamolodchikov A B 1986 JETP Lett. 43 730-732

[39] Zamolodchikov A B 1987 Sov. J. Nucl. Phys. 46 1090-1096

[40] Osborn H 1991 Nucl. Phys. B 363 486-526

[41] Chodos A et al 1974 Phys. Rev. D 93471

[42] Greiner W, Schramm S and Stein E 2002 Quantum Chromodynamics (Berlin: Springer-Verlag) 